

\title{
Term structure forecasts of long term consumption growth
}

\author{
Andrea Berardi \\ Università di Verona \\ Walter Torous \\ University of California at Los Angeles
}

April 2003

\begin{abstract}
Relying on a simple general equilibrium model of the term structure, both nominal yields and real consumption growth rates can be shown to be affine in the unobservable state variables. We can then express real consumption growth rates in terms of nominal yields rather than the unobservable state variables with the coefficients of the resultant forecasting relation being endogenously determined by the term structure model. As a result, we imply forecasts of real consumption growth rates from the term structure and provide empirical evidence consistent with our model more accurately predicting real consumption growth rates than a regression model based on the term spread.
\end{abstract}

${ }^{*}$ We would like to thank Stephen Schaefer for many helpful comments on an earlier draft of this paper. The comments of an anonymous referee and the editor, Jonathan Karpoff, significantly improved the paper. Any remaining errors, however, remain our responsibility. 


\section{Introduction}

This paper investigates the accuracy of using nominal interest rates to forecast real consumption growth rates and demonstrates that a simple general equilibrium model of the term structure provides more accurate forecasts than the simple term spread at horizons of one year and longer.

Beginning with Kessel (1965), many researchers including, among others, Harvey (1988, 1989, 1991 and 1993), Estrella and Hardouvelis (1991), Plosser and Rouwenhorst (1994), Chapman (1997), Kamara (1997), Roma and Torous (1997), and Hamilton and Kim (2002), have demonstrated that the term spread, that is, the difference between yields of long term and short term bonds, provides valuable predictive information about future economic growth. In particular, a positive term spread implies a subsequent increase in economic activity, while a negative term spread is consistent with a subsequent recession. The intuition for this result is based on the desire of investors to smooth consumption. For example, when a recession is expected, individuals will sell short term bonds and buy long term bonds to receive payoffs when their consumption level is expected to be lower. As a result, short term yields increase while long term yields decrease thereby inverting the yield curve in anticipation of a downturn in economic activity.

Distinct from the previous research, this paper investigates the link between interest rates and economic growth within a simple general equilibrium framework in which the behavior of both interest rates and real consumption growth are simultaneously modeled. Following Cox, Ingersoll, and Ross (1985), we construct a general equilibrium term structure model which imposes crossequation restrictions endogenously linking the term structure of interest rates to the dynamics of real consumption growth. Since both nominal yields and real consumption growth rates are affine in the posited but unobservable state variables, we can then express consumption growth rates in terms of nominal yields as opposed to the unobservable state variables.

As a result, we imply real consumption growth forecasts from the current nominal term structure and do not rely on consumption data to make our forecasts. Since today's yields on bonds maturing at different times in the future are set by investors taking into account the levels of consumption expected at those times, the forecasts we provide are forward looking. This 
contrasts to the spread model in which a historically estimated regression relying on past consumption growth rates specifies the forecasting relation. Unfortunately, the longer the forecast horizon, the more dated the estimated regression and so the less accurate are the spread model's forecasts when compared to our forecasts.

The plan of this paper is as follows. Section 2 details the general equilibrium model of the term structure and derives the endogenous relation between real consumption growth and nominal interest rates. In Section 3 we compare our forecasting model with a forecasting model based on the term spread and provide statistically reliable evidence that we more accurately forecast real consumption growth rates at horizons of one year and longer. We also statistically identify the sources of these forecast gains and document the robustness of our results to alternative specifications of the spread model. Section 4 concludes.

\section{The Model}

Our theoretical framework is based on the standard general equilibrium economy of the Cox, Ingersoll and Ross (1985) type. The main underlying assumptions are:

1. A fixed number of identical individuals with rational expectations maximizing a time-additive logarithmic utility function;

2. A competitive economy with continuous trading and no transactions costs;

3. The existence of markets for contingent claims and for instantaneous borrowing and lending at the riskless interest rate;

4. Production can be allocated to consumption or investment;

5. Investment opportunities consist of a stochastic production process, a set of contingent claims and a risk-free asset. 


\section{$2.1 \quad$ State Variables}

We assume that the economy is characterized by two latent state variables. Litterman and Scheinkman (1991) and Brown and Schaefer (1994) empirically document that the majority of the movement in the term structure of interest rates can be explained by two factors. In our case, these factors, $x$, follow risk-adjusted ${ }^{1}$ uncorrelated Gaussian processes:

$$
d x=(\phi+\Gamma x) d t+\Sigma d z
$$

where:

$x \equiv\left(\begin{array}{c}x_{1} \\ x_{2}\end{array}\right), \quad \phi \equiv\left(\begin{array}{c}\phi_{1} \\ \phi_{2}\end{array}\right), \quad \Gamma \equiv\left(\begin{array}{cc}\gamma_{1} & 0 \\ 0 & \gamma_{2}\end{array}\right), \quad \Sigma \equiv\left(\begin{array}{cc}\sigma_{1} & 0 \\ 0 & \sigma_{2}\end{array}\right)$.

The solution to this stochastic differential equation for $\tau>0$ gives:

$$
\begin{array}{r}
E_{t}\{x(t+\tau)\}=a(\tau)+B(\tau) x(t) \\
\operatorname{Cov}_{t}\left\{x(t+\tau), x(t+\tau)^{\prime}\right\}=F(\tau)
\end{array}
$$

where:

$$
a(\tau) \equiv \Gamma^{-1}(B(\tau)-\mathrm{I}) \phi, \quad B(\tau) \equiv \exp (\Gamma \tau), \quad F(\tau) \equiv-\left(\Psi-B(\tau) \Psi B(\tau)^{\prime}\right)
$$

and the matrix $\Psi$ is a function of the coefficients in matrices $\Gamma$ and $\Sigma .^{2}$

\subsection{Output and Consumption}

We assume that a single physical good is produced which may be allocated to consumption or investment and that a single technology exists allowing

\footnotetext{
${ }^{1}$ We directly specify the process for $x$ under the risk-adjusted probability measure by assuming that the parameter $\phi$ includes the risk-adjustment for the market price of risk. Doing so avoids the problem of identifying the market price of risk parameter when estimating contingent claims models based on Gaussian processes (see Dai and Singleton (2000)).

${ }^{2}$ See Langetieg (1980) footnote 22 for further details on the calculation of $\Psi$.
} 
capital to be transformed into output. Let $Q$ denote the nominal amount of the good invested in the production process and assume that it depends on both state variables. The following stochastic differential equation then describes the dynamics of nominal output in the economy:

$$
\frac{d Q}{Q}=\left(x_{1}+x_{2}\right) d t+\sigma_{Q} d z_{Q}
$$

Inflation in this economy is assumed to be non-stochastic. Let $p$ denote the price level and assume that it evolves according to the following deterministic process:

$$
\frac{d p}{p}=\pi d t
$$

where $\pi$ is the non-stochastic instantaneous expected inflation rate. ${ }^{3}$ Notice here that like Harvey (1988) we also assume a flat term structure of inflation expectations.

Applying Ito's lemma to the expression for real output, $q=Q / p$, allows us to derive a corresponding stochastic process for $q$. In equilibrium, all wealth will be invested in the production process and real consumption, $c$, must be proportional to optimally invested wealth ${ }^{4}, c=\delta W$. Therefore, a stochastic process of the following form holds for real consumption:

$$
\frac{d c}{c}=\left(x_{1}+x_{2}-\pi-\delta\right) d t+\sigma_{c} d z_{c}
$$

where $\sigma_{c}=\sigma_{Q}$ and $d z_{c}=d z_{Q}$.

Similarly, we can derive a stochastic differential equation for $\ln c$ and integrating this expression from $t$ to $t+\tau$ gives the following expression for the growth rate in consumption over the time interval $[t, t+\tau]$ :

\footnotetext{
${ }^{3}$ Assuming a stochastic inflation rate would result in a more complicated model which would also require us to hypothesize the nature of the interaction between inflation and output growth. Furthermore, not all of these additional parameters can be separately identified when estimating the resultant term structure model.

${ }^{4}$ See Cox, Ingersoll, and Ross (1985).
} 


$$
E_{t}\left\{\ln \frac{c(t+\tau)}{c(t)}\right\}=h(\tau ; \zeta)+J(\tau ; \zeta) x(t)
$$

with:

$$
\begin{aligned}
h(\tau ; \zeta) & =-\left(\delta+\pi+\frac{\sigma_{c}^{2}}{2}\right) \tau-\tau \iota^{\prime} \Gamma^{-1} \phi+J(\tau ; \zeta) \Gamma^{-1} \phi \\
J(\tau ; \zeta) & =\iota^{\prime} \Gamma^{-1}(B(\tau)-\mathrm{I}) \\
\iota^{\prime} & =\left(\begin{array}{ll}
1 & 1
\end{array}\right)
\end{aligned}
$$

where we now explicitly note the dependence upon all of the model's parameters $\zeta=\left(\phi_{1}, \phi_{2}, \gamma_{1}, \gamma_{2}, \sigma_{1}, \sigma_{2}, \sigma_{c}, \delta\right)$ which must be estimated.

\subsection{Term Structure of Interest Rates}

In equilibrium, the current time $t$ price of a nominal unit discount bond with maturity date $T=t+\tau$ is given by:

$$
G(t ; T)=E_{t}\left\{\frac{Q(t)}{Q(t+\tau)}\right\}
$$

Using standard results ${ }^{5}$, we can derive the following closed form solution for nominal bond prices:

$$
G(t ; T)=\exp \left[g_{0}(\tau ; \zeta)-g^{\prime}(\tau ; \zeta) x(t)\right]
$$

where

$$
\begin{aligned}
g_{0}(\tau ; \zeta) \equiv & \tau \sigma_{c}^{2}-\iota^{\prime} \Gamma^{-1}\left[\Gamma^{-1}(B(\tau)-\mathrm{I})-\tau \mathrm{I}\right] \phi \\
& +\frac{1}{2} g^{\prime}(\tau ; \zeta) \Psi g(\tau ; \zeta)+\frac{1}{2} \iota^{\prime} \Gamma^{-1} \Sigma \Sigma\left(\Gamma^{-1}\right)^{\prime} \iota \tau \\
& -\frac{1}{2} \iota^{\prime}\left[\Gamma^{-1} \Gamma^{-1}(B(\tau)-\mathrm{I}) \Psi+\Psi(B(\tau)-\mathrm{I})^{\prime}\left(\Gamma^{-1}\right)^{\prime}\left(\Gamma^{-1}\right)^{\prime}\right] \iota \\
g^{\prime}(\tau ; \zeta) \equiv & \iota^{\prime} \Gamma^{-1}(B(\tau)-\mathrm{I}) .
\end{aligned}
$$

\footnotetext{
${ }^{5}$ See, for example, Duffie (2001).
} 
Therefore, zero coupon yields can be expressed as:

$$
Y(t ; T) \equiv-\frac{\ln G(t ; T)}{T-t}=\kappa_{0}(\tau ; \zeta)+\kappa(\tau ; \zeta) x(t)
$$

where:

$$
\begin{aligned}
\kappa_{0}(\tau ; \zeta) & \equiv-g_{0}(\tau ; \zeta) / \tau \\
\kappa(\tau ; \zeta) & =\left(\kappa_{1}(\tau ; \zeta), \kappa_{2}(\tau ; \zeta)\right) \equiv g(\tau ; \zeta) / \tau
\end{aligned}
$$

\subsection{Implicit Relation Between Yields and Consump- tion}

Real consumption growth rates, expression (4), and nominal yields, expression (5), are both affine in the state variables. The closed form nature of these expressions implies that we can express consumption growth rates in terms of yields rather than in terms of the unobservable latent factors. Consequently, we provide an endogenous means of exploiting the nominal term structure to forecast real consumption growth rates. In other words, once we have estimated the nominal term structure model, we can simply imply the resultant real consumption growth rate forecasts.

To fix matters, we can express the two posited state variables in terms of two distinct yields, say the yield on a short term bond, $Y_{S} \equiv Y\left(\tau_{S}\right)$, and the yield on a long term bond, $Y_{L} \equiv Y\left(\tau_{L}\right), \tau_{L}>\tau_{S}$. Equivalently, to make our results comparable to previous forecasting models which rely on the spread, for example, Estrella and Hardouvelis (1991) and Harvey (1989, 1991, 1993), we can express the state variables in terms of the yield on a short term bond, $Y_{S}$, and the spread between long term and short term yields, $S P \equiv Y_{L}-Y_{S}$. From (5) we have:

$$
Z(t) \equiv\left(\begin{array}{c}
Y_{S}(t) \\
S P(t)
\end{array}\right)=\bar{\kappa}_{0}(\zeta)+\bar{\kappa}(\zeta) x(t)
$$

where:

$$
\begin{aligned}
& \bar{\kappa}_{0}(\zeta) \equiv\left(\begin{array}{c}
\kappa_{0}\left(\tau_{S} ; \zeta\right) \\
\kappa_{0}\left(\tau_{L} ; \zeta\right)-\kappa_{0}\left(\tau_{S} ; \zeta\right)
\end{array}\right), \\
& \bar{\kappa}(\zeta) \equiv\left(\begin{array}{cc}
\kappa_{1}\left(\tau_{S} ; \zeta\right) & \kappa_{2}\left(\tau_{S} ; \zeta\right) \\
\kappa_{1}\left(\tau_{L} ; \zeta\right)-\kappa_{1}\left(\tau_{S} ; \zeta\right) & \kappa_{2}\left(\tau_{L} ; \zeta\right)-\kappa_{2}\left(\tau_{S} ; \zeta\right)
\end{array}\right) .
\end{aligned}
$$


This system can be inverted with respect to the two latent state variables. Substituting the resulting expressions into expression (4) above, we can derive the endogenous relation between expected consumption growth, the spread and the short term yield:

$$
\begin{aligned}
E_{t}\left\{\ln \frac{c(t+\tau)}{c(t)}\right\} & =\alpha(\tau ; \zeta)+\beta(\tau ; \zeta) Z(t) \\
& =\alpha(\tau ; \zeta)+\beta_{1}(\tau ; \zeta) Y_{S}(t)+\beta_{2}(\tau ; \zeta) S P(t)
\end{aligned}
$$

where:

$\alpha(\tau ; \zeta) \equiv h(\tau ; \zeta)-\beta(\tau ; \zeta) \cdot \bar{\kappa}_{0}(\zeta), \quad \beta(\tau ; \zeta)=\left(\beta_{1}(\tau ; \zeta), \beta_{2}(\tau ; \zeta)\right) \equiv J(\tau ; \zeta) \cdot \bar{\kappa}^{-1}(\zeta)$

Expression (6) gives the consumption growth forecasting relation implied by the general equilibrium term structure model. By construction, this forecasting model depends on both the short term yield and the spread. More importantly, the coefficients on the short term yield, $\beta_{1}(\tau ; \zeta)$, and on the spread, $\beta_{2}(\tau ; \zeta)$, are endogenously determined and depend explicitly on the model's parameters $\zeta$. As a consequence, we do not use consumption data to construct our forecasts as all relevant information about future consumption growth is captured in general equilibrium by the term structure.

By comparison, forecasting models which rely on the spread are implemented as follows:

$$
E_{t}\left\{\ln \frac{c(t+\tau)}{c(t)}\right\}=\hat{a}(\tau)+\hat{b}(\tau) S P(t)
$$

where the parameters $\hat{a}(\tau)$ and $\hat{b}(\tau)$ are typically estimated from an in-sample regression of realized $\tau$-period growth rates onto past spreads. Unlike our two factor model, these parameters are not endogenously determined but rather depend on the historically estimated relation between real consumption growth rates and spreads. 


\section{Empirical Results}

In this section the two factor model, expression (6), is compared to the spread model, expression (7), in terms of their predictive accuracy in forecasting real consumption growth rates. To implement the two factor model requires that we fit the general equilibrium term structure model to prevailing yields and then construct forecasts at various horizons $\tau$ according to expression (6). Alternatively, the spread model uses the historically estimated relation between $\tau$ period real consumption growth rates and spreads to form $\tau$ period ahead forecasts according to (7).

Our subsequent empirical analysis relies on U.S. data drawn exclusively from the post-Volcker experiment era, 1985-2002. By doing so, we attempt to ensure that data are not sampled from differing macroeconomic regimes in which case we may erroneously attribute as forecast error a result which is due entirely to a change in macroeconomic regimes. ${ }^{6}$

\subsection{Data}

Term structure data used to estimate the model are monthly observations over the sample period 1985 to 2000 on the annualized zero coupon yields (the average of bid and ask yields) of U.S. Treasuries for six distinct maturities: three months and from one to five years. The three month data are taken from CRSP's Fama file while the one to five year data are taken from CRSP's Fama-Bliss file.

Our consumption data are monthly observations 1985 to 2002 on seasonally adjusted real (1996 dollars) personal expenditures on services plus nondurables from the U.S. Department of Commerce's Bureau of Economic Analysis. The corresponding deflator of personal expenditures on services plus non-durables measures the price level used to estimate the expected rate of inflation $\pi$ in expression (6). In particular, for each month we use the previous ten years of monthly observations on the logarithmic change in this deflator to fit an $A R I M A(1,0,1)$ model and take the resultant one-step ahead forecast as our estimate of $\pi$.

\footnotetext{
${ }^{6}$ See Chapman (1997) for extensive empirical evidence consistent with the choice of 1985 as the break point delineating the beginning of the post-Volcker experiment era.
} 


\subsection{Term Structure Model Estimation}

We cast the estimation of the general equilibrium term structure model in a linear state-space framework. ${ }^{7}$ Consistent with the model, the underlying state variable, $x(t)$, is explicitly recognized to be unobserved while observed bond yields are assumed to be a linear function of $x(t)$. While the term structure model is derived in continuous-time, its estimation will be carried out in discrete-time as yield data are only available at discrete time intervals of length $\Delta \equiv$ one month.

Suppose that at each date $t$ we observe yields of bonds with $M$ distinct maturity dates $T_{1}, T_{2} \ldots T_{M}$ or, equivalently, $M$ distinct terms to maturity, $\tau_{1}, \tau_{2} \ldots \tau_{M}, Y_{t}=\left(Y\left(t ; T_{1}\right), Y\left(t ; T_{2}\right) \ldots Y\left(t ; T_{M}\right)\right)^{\prime}$. Each observed yield can be expressed as the corresponding yield given by the model plus an independent, normally distributed measurement error, $e_{t, \tau_{i}}$. Measurement errors in the observed bond yields reflect noise arising from, for example, the bid-ask spread or possible quotation errors. This gives the following set of measurement equations:

$$
Y_{t}=K_{0}+K x_{t}+e_{t}
$$

where

$$
K_{0} \equiv\left(\begin{array}{c}
\kappa_{0}\left(\tau_{1} ; \zeta\right) \\
\cdots \\
\kappa_{0}\left(\tau_{M} ; \zeta\right)
\end{array}\right), \quad K \equiv\left(\begin{array}{c}
\kappa\left(\tau_{1} ; \zeta\right) \\
\ldots \\
\kappa\left(\tau_{M} ; \zeta\right)
\end{array}\right), \text { and } e_{t} \equiv\left(\begin{array}{c}
e_{t, \tau_{1}} \\
\cdots \\
e_{t, \tau_{M}}
\end{array}\right)
$$

In addition, the state variable's transition equation in discrete-time can be written as:

$$
x_{t+\Delta}=a(\Delta)+B(\Delta) x_{t}+v_{t}
$$

where the transition errors $v_{t}$ are assumed to be independently bivariate normally distributed with mean equal to the zero vector and covariance matrix given by $F(\Delta)$ from expression (3) which imposes cross-equation restrictions

\footnotetext{
${ }^{7}$ See Duffee (1999) and references therein on estimating term structure models in a state-space framework.
} 
on the variance and covariance properties of the state variables. To complete the specification, the measurement errors $e_{t}$ and the transition errors $v_{t}$ are assumed to be uncorrelated at all lags and to be uncorrelated with the initial state vector.

With these assumptions, we may use the Kalman filter to optimally predict the underlying state variable, $x_{t}$, as well as to efficiently evaluate the corresponding likelihood function. Numerical optimization of this likelihood function over $\zeta$ gives the maximum likelihood estimator $\hat{\zeta}$ of the parameters of the general equilibrium term structure model. ${ }^{8}$

\subsubsection{Term Structure Model Estimation Results}

We recursively estimate the general equilibrium term structure model using a fixed ten year window of monthly data beginning in February 1985. That is, using one hundred and twenty months of yield data from February 1985 to January 1995, we fit the term structure model as of January 1995, obtain the corresponding maximum likelihood parameter estimates and measure the errors in pricing the sampled Treasury securities through January 1995. Subsequently, moving forward one month, the one hundred and twenty months of yield data ending in February 1995 allow us to update the maximum likelihood estimates and measure the errors in fitting the term structure through February 1995. Proceeding recursively in this fashion, we obtain maximum likelihood estimates of the term structure model's parameters at monthly intervals from January 1995 to December 2000 as well as corresponding Treasury pricing errors.

The term structure model's maximum likelihood parameter estimates from January 1995 through December 2000 are summarized in Table $1 .{ }^{9}$ Notice that the estimated mean reversion coefficients, $\hat{\gamma}_{1}$ and $\hat{\gamma}_{2}$, are, on average,

\footnotetext{
${ }^{8}$ We do not estimate the parameter $\delta$, the rate of patience, because this parameter does not enter the closed form solution for yields given by expression (5). However, as other studies, for example, Dunn and Singleton (1986) and Ferson and Constantinides (1991), have found the intertemporal coefficient $\beta=e^{-\delta}$, a one-to-one transformation of $\delta$, to be statistically indistinguishable from one, we estimate the remaining parameters under the equivalent restriction that $\delta$ equals zero. Our results do not change qualitatively if we set $\beta$ to be less than but close to one or, equivalently, we set $\delta$ to be greater than but close to zero.

${ }^{9}$ Given the recursive nature of our estimation procedure, these parameter estimates
} 
consistent with the first factor behaving like a random walk, $\hat{\gamma}_{1} \approx 0$, while the second factor is more stationary in its behavior, $\hat{\gamma}_{2}<0$. Despite this difference, the corresponding estimated volatility coefficients, $\hat{\sigma}_{1}$ and $\hat{\sigma}_{2}$, are quite similar on average.

Summary statistics for the resultant errors in pricing the sampled zero coupon Treasury yields are provided in Table 2. An error here is defined as the fitted yield minus the actual yield and is measured in basis points. To interpret these statistics recall that in fitting the term structure model we have measured these errors by maturity for each of the preceeding one hundred and twenty months of sampled yield data. For each estimation date, we can then calculate the resultant mean errors and root mean squared errors. Table 2 provides the average of these errors across all of the estimation dates. ${ }^{10}$ The results of Table 2 indicate that the model provides an adequate fit to the term structure although it does not fit the short-end as well.

\subsection{Forecasting Results}

To forecast real consumption growth using the two factor model, we use the preceeding ten years of yield data to estimate the parameters of the general equilibrium model needed in expression (6) to forecast three and six months ahead as well as one through five years ahead. Proceeding recursively in this fashion from January 1995 through December 2000, we compute consumption growth forecasts which are then compared to realized consumption growth rates.

Alternatively, to forecast real consumption growth using the spread model, expression (7), we use the preceeding ten years of yield and real consumption data to fit linear regressions of realized consumption growth rates against the spread observed between five year and three month yields. Linear regressions are separately fit for each of the forecast horizons. The corresponding estimated coefficients are then used to forecast consumption growth rates over

are not independent. Because our focus is on investigating the predictive accuracy of consumption growth forecasts, we do not provide a statistical analysis of these parameter estimates which takes their overlapping nature into account. Any serial dependence in the resultant consumption growth forecasts, however, will be explicitly taken into account.

${ }^{10}$ Once again, given the recursive nature of our estimation procedure, these errors are not independent and the summary statistics are provided for illustrative purposes. 
that particular horizon. Proceeding recursively from January 1995 through December 2000 gives competing forecasts to those produced using our general equilibrium model.

Average differences between the spread model's squared errors and the two factor model's squared errors in forecasting realized consumption growth rates are tabulated in Table 3. A positive difference here is consistent with the two factor model being more accurate. ${ }^{11}$ We also present the results of the Diebold and Mariano (1995) test of the null hypothesis of no difference in the accuracy of these competing forecasts. ${ }^{12}$

The clear message that emerges from Table 3 is that the two factor model provides more accurate forecasts of real consumption growth rates at forecasting horizons of one year and longer. At short horizons, three or six months, we cannot reject the null hypothesis that there is no difference in the accuracy of the competing forecasts. At longer horizons, however, we see reliable evidence that the two factor model's forecasts are more accurate than the spread model's forecasts.

These conclusions are reinforced graphically in Figure 1. Both forecasting models are least accurate at short horizons where real consumption growth rates are extremely noisy. As expected, as the forecast horizon lengthens, the accuracy of both models tends to improve. The improvement in the forecast accuracy of the spread model, however, is less dramatic. For example, at three, four, and five year horizons, the two factor model's forecasts

\footnotetext{
${ }^{11}$ Qualitatively similar results obtain for the corresponding absolute errors and are not reported here.

${ }^{12}$ Let $d_{t}$ denote the difference in squared errors between the competing forecasts at $t$ or, in other words, the loss differential at $t$. Under the null hypothesis that the population mean of the loss differential series $\left\{d_{t}\right\}$ is zero, the statistic $\bar{d} / \sqrt{2 \pi \hat{f}_{d}(0)} T$ is asymptotically standard normal distributed where $\hat{f}_{d}(0)$ is a consistent estimate of the spectral density of the loss differential at frequency $\omega=0$. Following Newey and West (1987), a consistent estimate of $2 \pi f_{d}(0)$ is obtained by using a Bartlett kernel with lag selected according to Newey and West's (1994) automatic bandwidth selection procedure. As noted by Diebold (2001), the Diebold-Mariano statistic is simply a $t$-statistic for the hypothesis of a zero population mean loss differential, adjusted to reflect the fact that the loss differential series is not necessarily white noise. In practice, we compute the Diebold-Mariano statistic by simply regressing the appropriate loss differential series against an intercept and correcting this equation for serial correlation.
} 
are within approximately fifty basis points of corresponding actual real consumption growth rates whereas the spread model's are within one hundred or more basis points. To understand this, recall that as the forecast horizon lengthens, the spread model relies on more dated information. For example, using the spread model to forecast five year growth rates implies that the latest observation available to estimate the model is the spread prevailing five years ago together with the subsequent five year growth rate. The use of anachronous data is not a problem with our two factor model as we do not rely on consumption data whatsoever, rather we imply this forecast from today's term structure. ${ }^{13}$

To further investigate the two factor model, Figure 2 graphically displays the time series properties of the model's forecasting errors, defined as predicted real consumption growth rates minus observed growth rates. Panel A of Figure 3 considers three, six, and twelve month forecasting horizons, while Panel B considers two, three, four and five year horizons. While the forecasts appear to track real consumption growth rates fairly well over the entire sample period, the model does appear to systematically under predict real consumption growth during the late 1990s, especially at short horizons (Panel A). One interpretation of this result is that actual consumption growth rates were unexpectedly high here, at least relative to what was being predicted by the term structure of interest rates, because of the significant stock market appreciation surrounding the Internet bubble and the consequent effects of this increase in stock market wealth on consumer spending. ${ }^{14}$

\subsubsection{Sources of Improved Forecast Accuracy}

While the results of Table 3 are consistent with the two factor model giving more accurate forecasts at most horizons, no information is provided as to whether this improvement is due to lower bias, lower variance, or both. We

\footnotetext{
${ }^{13}$ To further emphasize this, in unreported results, we also fit a one factor version of our model and implied corresponding real consumption growth rates. While the resultant short horizon forecasts are less accurate, the long horizon forecasts are still more accurate than those of the spread model. We also explored a three factor version of our model but as it offers but a modest improvement in fitting prevailing yield curves, it does not provide statistically more accurate consumption forecasts than the two factor model.

${ }^{14}$ See, for example, Poterba (2000) for an investigation of the effects of stock market wealth on consumption.
} 
can, however, test for the significance of these sources of improvement by following Ashley, Granger, and Schmalensee (1980) whose test procedures are applicable even if the forecast errors are cross-correlated, autocorrelated and have non-zero means.

In particular, letting $e_{1, t}$ and $e_{2, t}$ denote the forecast errors of the spread model and the two factor model, respectively, if $\Delta_{t}$ denotes the difference in these forecast errors, $\Delta_{t} \equiv e_{1 t}-e_{2 t}$, and $\Sigma_{t}$ denotes their sum, $\Sigma_{t} \equiv e_{1 t}+e_{2 t}$, then the regression equation

$$
\Delta_{t}=\beta_{0}+\beta_{1}\left(\Sigma_{t}-\bar{\Sigma}\right)+u_{t}
$$

allows us to test the null hypotheses that there is no difference in the biases of the competing forecasts, $H_{0}: \beta_{0}=0$, and that there is no difference in the variances of the competing forecasts, $H_{0}: \beta_{1}=0 .{ }^{15}$

The results are tabulated in Table 4. There for each horizon we decompose the difference in mean squared forecasting errors of the spread model versus the two factor model into the corresponding differences in squared mean errors and variance of their forecasting errors together with the statistical significance of these differences.

At horizons of one year and longer, the two factor model's forecasts are significantly less biased than those of the spread model. Furthermore, the two factor model's forecasts are significantly less variable at the two year horizon but more variable at horizons of four and five years. In general, the results of Table 4 point to the improvement in the two factor model's mean squared forecasting errors stemming from their consistently smaller mean forecasting errors.

\footnotetext{
${ }^{15}$ Since the sample mean squared error, $M S E$, can be decomposed into the sample variance, $s^{2}$, plus the sample mean error squared, $m^{2}$, we can write $M S E\left(e_{1}\right)-M S E\left(e_{2}\right)=$ $\left[s^{2}\left(e_{1}\right)-s^{2}\left(e_{2}\right)\right]+\left[m\left(e_{1}\right)^{2}-m\left(e_{2}\right)^{2}\right]$, which with further manipulation can be expressed as $M S E\left(e_{1}\right)-M S E\left(e_{2}\right)=\widehat{\operatorname{cov}}(\Delta, \Sigma)+\left[m\left(e_{1}\right)^{2}-m\left(e_{2}\right)^{2}\right]$ where $\widehat{\operatorname{cov}}$ denotes sample covariance. The regression equation (10), where $u_{t}$ is a mean zero error term assumed to be independent of $\Sigma$, and the corresponding tests immediately follow. Without loss of any generality, the discussion here and in the text assumes that both error means are positive. The methodology can be easily generalized to the other cases.
} 


\subsubsection{Using the Entire Term Structure of Spreads}

Until now, the empirical analysis of the spread model has relied on just two points on the term structure, the five year and three month yields, to forecast real consumption growth rates at all horizons. By comparison, the two factor model uses information from all of the sampled yields. Therefore, the improvement in the two factor model's forecasting accuracy may simply reflect the fact that it makes use of the entire term structure not just two points on it.

To investigate whether this is the case, we can compare the two factor model to a system of spread regressions in which each regression relies on the spread calculated over that particular portion of the term structure corresponding to the horizon over which real consumption growth is being forecasted. That is, we use the spread between $\tau$-period and one month yields to forecast the $\tau$-period real consumption growth rate, where $\tau=$ three months, six months, one year, two years, three years, four years, and five years. ${ }^{16}$ This system of regressions allows the spread model to take advantage of all of the sampled yields not just two.

These results are given in Table 5. While the forecast accuracy of the spread model is now improved, it still is the case that the two factor model is more accurate at longer horizons.

\section{Conclusions}

Investors set the yields of bonds maturing at different times in the future by taking into account the levels of consumption expected at those times. In this paper, we recover these investor expectations from a simple general equilibrium model of the term structure. By fitting this model to observed yields, we are able to imply ex ante forecasts of real consumption growth without relying on consumption data whatsoever. This is in contrast to the spread model whose forecasts are obtained by extrapolating the ex post relation between the term spread and subsequent realized consumption growth.

\footnotetext{
${ }^{16}$ Like the three month Treasury yield, the one month Treasury yield is taken from CRSP's Fama file. The six month yield is taken from the FRED database of the Federal Reserve Bank of St. Louis.
} 
By very construction, the longer the forecast horizon, the more dated this relation and so the less accurate the spread model's forecasts. Our empirical results are indeed consistent with the increased predictive accuracy of our general equilibrium approach. 


\section{References}

1. Ashley, R., C.W.J. Granger, and R. Schmalensee (1980): "Advertising and aggregate consumption: an analysis of causality", Econometrica, 48, 1149-1167.

2. Breeden, D.T. (1986): "Consumption, production, inflation and interest rates: a synthesis", Journal of Financial Economics, 16, 3-39.

3. Brown, R. H. and S.M. Schaefer (1994): "Interest rate volatility and the shape of the term structure", Philosophical Transactions of the Royal Society A, 347, 563-576.

4. Chapman, D.A. (1997): "The cyclical properties of consumption growth and the real term structure", Journal of Monetary Economics, 39, 145172 .

5. Cox, J.C., J.E. Ingersoll and S.A. Ross (1985): "An intertemporal general equilibrium model of asset prices", Econometrica, 53, 363-384.

6. Dai, Q. and K.J. Singleton (2000): "Specification analysis of affine term structure models", Journal of Finance, 55, 1943-1978.

7. Diebold, F.X. (2001): Elements of Forecasting. Second edition. Cincinnati, $\mathrm{OH}$ : South-Western Publishing.

8. Diebold, F.X. and R.S. Mariano (1995): "Comparing predictive accuracy", Journal of Business and Economic Statistics, 13, 253-63.

9. Duffee, G.R. (1999): "Estimating the price of default risk", Review of Financial Studies, 12, 197-226.

10. Duffie, D. (2001): Dynamic asset pricing theory. Third edition. Princeton, N.J.: Princeton University Press.

11. Dunn, K.B. and K.J. Singleton (1986): "Modeling the term structure of interest rates under non-separable utility and durable goods", Journal of Financial Economics, 17, 27-55. 
12. Estrella, A. and G.A. Hardouvelis (1991): "The term structure as a predictor of real economic activity", Journal of Finance, 46, 555-576.

13. Ferson, W.E. and G.M. Constantinides (1991): "Habit persistence and durability in aggregate consumption: empirical tests", Journal of Financial Economics, 29, 199-240.

14. Hamilton, J.D. and D.H. Kim (2002): "A re-examination of the predictability of economic activity using the yield spread", Journal of Money, Credit and Banking, 34, 340-360.

15. Harvey, C.R. (1988): "The real term structure and consumption growth", Journal of Financial Economics, 22, 305-334.

16. Harvey, C.R. (1989): "Forecasts of economic growth from the bond and stock markets", Financial Analysts Journal, September/October, $38-45$.

17. Harvey, C.R. (1991): "The term structure and world economic growth", Journal of Fixed Income, 1, 4-17.

18. Harvey, C.R. (1993): "Term structure forecasts economic growth", Financial Analysts Journal, May/June, 6-8.

19. Kamara, A. (1997): "The relation between default-free interest rates and expected economic growth is stronger than you think", Journal of Finance, 52, 1681-1694.

20. Kessel, R. (1965): "The cyclical behavior of the term structure of interest rates", occasional paper no. 91, National Bureau of Economic Research, Cambridge, Mass.

21. Langetieg, T.C. (1980): "A multivariate model of the term structure", Journal of Finance, 35, 71-97.

22. Litterman, R. and J. Scheinkman (1991): "Common factors affecting bond returns", Journal of Fixed Income, 1, 54-61.

23. Newey, W. and K. West (1987): "A simple positive semi-definite, heteroskedasticity and autocorrelation consistent covariance matrix", Econometrica, 55, 703-708. 
24. Newey, W. and K. West (1994): "Automatic lag selection in covariance matrix estimation", Review of Economic Studies, 61, 631-653.

25. Plosser, C.I. and K.G. Rouwenhorst (1994): "International term structures and real economic growth", Journal of Monetary Economics, 33, $133-155$.

26. Poterba, J. (2000): "Stock Market Wealth and Consumption", Journal of Economic Perspectives, 14, 99-118.

27. Roma, A. and W. Torous (1997): "The cyclical behavior of interest rates", Journal of Finance, 52, 1519-1542. 
Table 1

\section{Term Structure Model's Parameter Estimates}

This table summarizes the term structure model's parameter estimates obtained by fitting zero coupon Treasury yields. We estimate the model using maximum likelihood by casting it in a discrete-time state-space framework and evaluating the likelihood function using the Kalman filter. Proceeding recursively, we estimate the term structure model at monthly intervals from February 1995 to December 2000.

\begin{tabular}{|c|ccccccc|}
\hline parameter estimate & $\hat{\gamma}_{1}$ & $\hat{\gamma}_{1}$ & $\hat{\phi}_{1}$ & $\hat{\phi}_{2}$ & $\hat{\sigma}_{1}$ & $\hat{\sigma}_{2}$ & $\hat{\sigma}_{c}$ \\
\hline mean & -0.0746 & -0.8990 & 0.0059 & 0.0140 & 0.0159 & 0.0153 & 0.0031 \\
standard deviation & 0.0701 & 0.2000 & 0.0057 & 0.0147 & 0.0041 & 0.0046 & 0.0097 \\
\hline
\end{tabular}




\section{Table 2}

\section{Summary Statistics of Term Structure Model's Pricing Errors}

This table summarizes the term structure model's error properties in fitting zero coupon Treasury yields over the sample period 1985:2 to 2000:12. An error is defined as a fitted yield minus an observed yield and is measured in basis points. Yield data, the average of bid and ask, are obtained from CRSP's Fama file (three month maturity) and CRSP's Fama-Bliss file (one year through five years). We estimate the model using maximum likelihood by casting it in a discrete-time state-space framework and evaluating the likelihood function using the Kalman filter. Proceeding recursively, we estimate the term structure model at monthly intervals from January 1995 to December 2000 and at each date measure the resultant errors in fitting the preceeding ten year's of monthly yields.

\begin{tabular}{|l|c|c|c|}
\hline Yield maturity & $\begin{array}{c}\text { Average of } \\
\text { Mean Errors } \\
(\text { bps })\end{array}$ & $\begin{array}{c}\text { Standard Deviation of } \\
\text { Mean Errors } \\
(\text { bps })\end{array}$ & $\begin{array}{c}\text { Average of Root } \\
\text { Mean Squared Errors } \\
(\text { bps })\end{array}$ \\
\hline 3 months & 2.70 & 6.07 & 17.83 \\
1 year & -3.68 & 2.39 & 15.80 \\
2 years & 1.15 & 2.28 & 12.41 \\
3 years & 2.00 & 2.52 & 7.19 \\
4 years & -1.72 & 2.60 & 8.09 \\
5 years & -0.45 & 3.58 & 12.25 \\
\hline
\end{tabular}




\section{Table 3}

\section{Comparing Predictive Accuracy in Forecasting Real Consumption Growth Rates: The Two Factor Model versus the Spread Model using the Spread between Five Year and Three Month Yields at all Horizons.}

This table compares the predictive accuracy of the two factor model versus the spread model in forecasting real consumption growth rates. The spread model uses the spread between five year and three month yields throughout. Predictive accuracy is measured by a model's corresponding squared error. We tabulate by forecast horizon the average across estimation dates of the differences between the spread model's and the two factor model's squared errors. The asymptotic $p$-values of the Diebold-Mariano (1995) statistic testing the null hypothesis of no difference in the accuracy of these competing forecasts are also provided.

\begin{tabular}{|l|cc|}
\hline $\begin{array}{l}\text { Time } \\
\text { Horizon }\end{array}$ & $\begin{array}{c}\text { Average of Differences } \\
\text { in Squared Errors }\end{array}$ & $\begin{array}{c}p \text {-value of } \\
\text { Diebold-Mariano Statistic }\end{array}$ \\
\hline 3 months & -0.18 & 0.85 \\
6 months & 0.69 & 0.49 \\
1 year & 2.30 & 0.02 \\
2 years & 3.96 & $<0.01$ \\
3 years & 4.23 & $<0.01$ \\
4 years & 6.54 & $<0.01$ \\
5 years & 4.15 & $<0.01$ \\
\hline
\end{tabular}




\section{Table 4}

\section{Sources of Improved Forecast Accuracy}

This table decomposes the differences in the sample mean squared forecasting errors $(M S E)$ of the spread model, with errors $e_{1, t}$, and the two factor model, with errors $e_{2, t}$, into the differences in their sample mean squared errors, $\mathrm{m}^{2}$, plus the differences in their sample variances, $s^{2}$ :

$$
M S E\left(e_{1}\right)-M S E\left(e_{2}\right)=\left[m\left(e_{1}\right)^{2}-m\left(e_{2}\right)^{2}\right]+\left[s^{2}\left(e_{1}\right)-s^{2}\left(e_{2}\right)\right] .
$$

We also tabulate the $p$-values of Ashley, Granger, and Schmalensee's (1980) tests of the null hypotheses that there is no difference in the biases of the competing forecasts and that there is no difference in the variances of the competing forecasts. The Newey and West (1987) correction for heterokedasticity and serial correlation is applied to take into account the overlapping nature of these forecasts.

\begin{tabular}{|l|cccc|}
\hline $\begin{array}{l}\text { Time } \\
\text { Horizon }\end{array}$ & $\begin{array}{c}\text { Differences in Sample } \\
\text { Mean Squared Errors }\end{array}$ & $\begin{array}{c}\text { Differences in Sample } \\
\text { Variances }\end{array}$ & $\begin{array}{c}p \text {-value of statistic } \\
\text { testing no difference } \\
\text { in biases }\end{array}$ & $\begin{array}{c}p \text {-value of statistic } \\
\text { testing no difference } \\
\text { in variances }\end{array}$ \\
\hline 3 months & -0.15 & 0.08 & 0.30 & 0.74 \\
6 months & 0.04 & 0.15 & 0.75 & 0.44 \\
1 year & 0.29 & 0.30 & 0.02 & 0.11 \\
2 years & 0.59 & 0.62 & $<0.01$ & $<0.01$ \\
3 years & 1.11 & 0.11 & $<0.01$ & 0.32 \\
4 years & 1.23 & -0.26 & $<0.01$ & $<0.01$ \\
5 years & 0.89 & -0.25 & $<0.01$ & $<0.01$ \\
\hline
\end{tabular}




\section{Table 5}

\section{Comparing Predictive Accuracy in Forecasting Real Consumption Growth Rates: The Two Factor Model versus the Spread Model using the Spread between $\tau$-Period and Three Month Yields at the $\tau$-Period Horizon.}

This table compares the predictive accuracy of the two factor model versus the spread model in forecasting real consumption growth rates. The spread model uses the spread between $\tau$-period and three month yields to forecast $\tau$ period growth rates where $\tau=$ three months, six months, one year, two years, three years, four years, and five years. Predictive accuracy is measured by a model's corresponding squared error. We tabulate by forecast horizon the average across estimation dates of the differences between the spread model's and the two factor model's squared errors. The asymptotic $p$-values of the Diebold-Mariano (1995) statistic testing the null hypothesis of no difference in the accuracy of these competing forecasts are also provided.

\begin{tabular}{|l|cc|}
\hline $\begin{array}{l}\text { Time } \\
\text { Horizon }\end{array}$ & $\begin{array}{c}\text { Average of Differences } \\
\text { in Squared Errors }\end{array}$ & $\begin{array}{c}p \text {-value of } \\
\text { Diebold-Mariano Statistic }\end{array}$ \\
\hline 3 months & -1.46 & 0.15 \\
6 months & -0.91 & 0.37 \\
1 year & 0.53 & 0.60 \\
2 years & 3.10 & $<0.01$ \\
3 years & 3.81 & $<0.01$ \\
4 years & 5.84 & $<0.01$ \\
5 years & 4.19 & $<0.01$ \\
\hline
\end{tabular}




\section{Figure 1}

\section{Mean Absolute Errors in Forecasting Consumption Growth Rates}

This figure compares the predictive accuracy of the two factor term structure model with the spread model in forecasting real consumption growth rates. Predictive accuracy is measured by a model's corresponding mean absolute error and is measured in basis points. The two factor model forecasts are obtained by using the preceeding ten years of yield data to estimate the parameters needed to forecast subsequent real consumption growth rates. Alternatively, to forecast real consumption growth using the term spread model, we use the preceeding ten years of yield and real consumption data to fit linear regressions of realized consumption growth rates against the spread observed between five year and three month yields. The corresponding estimated coefficients are then used to forecast subsequent consumption growth rates over the different horizons. Proceeding recursively, we compute real consumption growth forecasts for both competing models from December 1993 to December 1999 and compare them to realized consumption growth rates. 


\section{Figure 2}

\section{Two Factor Model's Consumption Forecasting Errors}

This figure shows the time series properties of the two factor model's forecasting errors, defined as predicted minus observed real consumption growth

rates. Model forecasts are obtained by using the preceeding ten years of yield data to estimate the parameters needed to forecast real consumption growth rates. Proceeding recursively, we compute consumption growth forecasts from December 1993 to December 1999 and compare them to realized consumption growth rates.

Panel A: Three, six and twelve month forecasting horizons:

Panel B: Two, three, four and five year forecasting horizons: 